\section{COVID-19: Impfung für Rheumapatienten auch unter der Behandlung sicher und wirksam}

Menschen mit rheumatischen Erkrankungen können und sollten sich gegen COVID-19 impfen lassen. Zwei in Deutschland durchgeführte Studien zeigen, dass die Impfung mit einer $\mathrm{mR}$ NA-Vakzine für Menschen mit Rheuma sicher ist und in der Regel gut vertragen wird. Auch kann nach Einschätzung der Deutschen Gesellschaft für Rheumatologie e.V. (DGRh) von einer guten Schutzwirkung ausgegangen werden. Jedenfalls legen das die Messungen der schützenden Antikörper nahe, die nach der Impfung von fast allen Rheumapatienten gebildet werden.

Viele Menschen mit rheumatischen Erkrankungen haben ein erhöhtes Infektionsrisiko. Dies betrifft insbesondere Patienten mit einer durch sogenannte Basistherapeutika nicht ausreichend kontrollierten Erkrankung und bei längerfristiger und höher dosierter Behandlung mit Kortisonpräparaten. Bei einer Infektion mit SARS-CoV-2 tragen diese Rheumapatienten ein erhöhtes Risiko, schwer an COVID-19 zu erkranken. Deshalb wurden Patienten mit rheumatischen Erkrankungen in der Impfreihenfolge der Gruppe 3 zugeordnet und können sich priorisiert impfen lassen. Unter Patienten und Ärzten gibt es jedoch Bedenken, berichtet DGRh-Präsident Prof. Andreas Krause. „Viele sind in Sorge, dass die mit der Impfung verbundene Immunreaktion einen Krankheitsschub auslösen könnte“, erklärt der Experte, der als ärztlicher Direktor der Klinik für Innere Medizin am Immanuel Krankenhaus Berlin tätig ist. Verbreitet sei auch die Befürchtung, dass die das Immunsystem beeinflussenden Medikamente, die viele Rheumapatienten erhalten, die Bildung von schützenden Antikörpern gegen SARS-CoV-2 verhindern. Die bisherigen Erfahrungen mit mRNA-Impfstoffen widersprechen dem jedoch, betont Prof. Krause.

Rheumatologen vom Campus Kiel des Universitätsklinikums Schleswig-Holstein haben in den letzten Wochen 26 Patienten mit verschiedenen rheumatischen Erkrankungen gegen COVID-19 geimpft. Zum Einsatz kamen die mRNA-Impfstoffe von Biontech/Pfizer oder Moderna. Die meisten Rheumapatienten wurden mit sogenannten Biologika behandelt, die das Fortschreiten der Erkrankung, etwa die Zerstörung der Gelenke, verhindern. Andere erhielten zu diesem Zweck herkömmliche Basistherapeutika oder Kortison. „Diese Mittel hemmen das Immunsystem. Sie könnten deshalb im Prinzip die Bildung von Antikörpern oder Abwehrzellen gegen SARS-CoV-2 verhindern", berichtet Studienleiterin Prof. Bimba Hoyer, die deshalb genaue Tests zur Immunreaktion durchführen ließ. Der Vergleich mit 42 gesunden Menschen ergab, dass die Antikörper-Reaktion tatsächlich etwas schwächer ausfiel. In einem Test wurden $2053 \mathrm{BAU} / \mathrm{ml}$ gemessen gegenüber 2685 bei den gesunden Kontrollen. BAU steht für „binding antibody units“ und ist ein Maß für die Menge der Antikörper in Blut. Trotz der leicht verminderten Antikörper-Antwort dürften die meisten Patienten nach Einschätzung von Hoyer vor COVID19 geschützt sein oder wenigstens ein schwerer Verlauf verhindert werden. „Keiner unserer Patienten war ein kompletter Non-Responder und damit ein sogenannter „Impfversager'“, berichtet die Rheumatologin. Von einem Absetzen der Rheumamittel, um damit die Impfwirkung zu verbessern, rät die Expertin ab. Dies könne einen Krankheitsschub auslösen, der die Situation der Patienten verschlechtert. Die Impfung hat sich auch nicht negativ auf den rheuma- 
tologischen Krankheitsverlauf ausgewirkt: „Bei keinem Patienten kam es zu einer Verstärkung der Beschwerden oder einem Krankheitsschub.

Auch das Team um Prof. Georg Schett vom Universitätsklinikum Erlangen hat gute Erfahrungen mit der Impfung von Rheumapatienten gemacht. Im Rahmen einer Studie des Deutschen Zentrums für Immuntherapie haben in Erlangen 84 Patienten den Impfstoff von Biontech/Pfizer erhalten. Die Antikörper-Antwort auf die Impfung sowie die neutralisierende Wirkung der Antikörper fiel bei Patienten mit rheumatoider Arthritis, Psoriasis Arthritis, Spondyloarthritis und Kollagenosen etwas schwächer aus als in einer Kontrollgruppe von 182 gesunden Personen. Die Verabreichung von Biologika beeinflusste dabei die Immunantwort auf die Impfung nicht. Bezüglich der wenigen Patienten, die keine Antikörper bildeten, meint Schett, dass „diese Patienten nicht notwendigerweise ungeschützt sind, sondern möglicherweise eine zelluläre Immunantwort gegen das Virus bilden und durch diese zumindest teilweise geschützt sein könnten“.

Nach einer Pressemitteilung der Deutsche Gesellschaft für Rheumatologie 